\title{
Ground states of the massless Derezinśki-Gerard model
}

$\operatorname{AUTHOR}(S)$ :

Ohkubo, Atsushi

\section{CITATION:}

Ohkubo, Atsushi. Ground states of the massless Derezinski-Gerard model. Journal of Mathematical Physics 2009, 50(11): 113511.

\section{ISSUE DATE:}

2009

URL:

http://hdl.handle.net/2433/91264

RIGHT:

c 2009 American Institute of Physics. 


\title{
Ground states of the massless Dereziński-Gérard model
}

\author{
Atsushi Ohkubo a) \\ Graduate School of Informatics, Kyoto University, Yoshida-Honmachi, Sakyo-ku, \\ Kyoto 606-8501, Japan
}

(Received 27 December 2008; accepted 1 October 2009; published online 13 November 2009)

\begin{abstract}
We consider the massless Dereziński-Gérard model introduced by Dereziński and Gérard in 1999. We give a sufficient condition for the existence of a ground state of the massless Dereziński-Gérard model without the assumption that the Hamiltonian of particles has compact resolvent. (C) 2009 American Institute of Physics. [doi:10.1063/1.3253976]
\end{abstract}

\section{INTRODUCTION}

The Dereziński-Gérard model was introduced by Dereziński and Gérard ${ }^{5}$ as an abstract model of particle-field interaction (they call it the Pauli-Fierz model, but we call it the DerezińskiGérard model for the sake of clarity.). The Hamiltonian of the Dereziński-Gérard model is given by

$$
H:=A \otimes I+I \otimes H_{\mathrm{b}}+\widetilde{\phi}(v) .
$$

The first and second terms mean Hamiltonians of particles and a quantum field, respectively. The third term means the interaction between particles and a quantum field.

The existence of a ground state of the massive Dereziński-Gérard model was investigated by Dereziński and Gérard ${ }^{5}$ and Miyao and Sasaki. ${ }^{10}$ Dereziński and Gérard ${ }^{5}$ showed the existence of a ground state of the massive Dereziński-Gérard model by specifying the essential spectrum of the Hamiltonian, which is an analog of the HVZ theorem in many body problems. Miyao and Sasaki ${ }^{10}$ showed the existence of a ground state of the massive Dereziński-Gérard model by applying the method developed by Arai and Hirokawa. ${ }^{2}$

In Ref. 7, Georgescu et al. discussed spectral analysis by means of Mourre estimate, limiting absorption principle, and local finiteness of eigenvalues in the massless Dereziński-Gérard model. In Ref. 8, Gérard showed the existence of a ground state of the massless Dereziński-Gérard model under the assumption that the Hamiltonian of particles $A$ has compact resolvent. In Ref. 4, Bruneau and Dereziński extended the results of Refs. 5 and 8 by using quadratic form techniques under the same assumption as that of Ref. 8.

In the present paper, we consider the same problem as that of Ref. 8 without the above assumption. This is a big difference between Refs. 8 and 4 and the present paper.

The present paper is organized as follows. In Sec. II we describe the model considered in this paper and state the main result. In Sec. III, we first prove an energy bound for the DerezińskiGérard model. Second, we state commutation relations for the generalized annihilation/creation operators and relative boundedness of them. Finally, we prove the self-adjointness of the total Hamiltonian. Section IV is a preliminary to Sec. V. In Sec. IV, we prove some estimates which are used to prove the existence of a ground state of the massless Dereziński-Gerrard model. Section V is devoted to the proof of the existence of a ground state of the massless Dereziński-Gérard model.

\footnotetext{
a)Eletronic mail: ohkubo@acs.i.kyoto-u.ac.jp.
} 


\section{DEFINITION OF THE MODEL}

In the present paper, we denote the inner product and the norm of a Hilbert space $\mathcal{X}$ by $\langle\cdot, \cdot\rangle_{\mathcal{X}}$ and $\|\cdot\|_{\mathcal{X}}$, respectively. The inner product is antilinear in the first variable. If there is no danger of confusion, then we omit the subscript $\mathcal{X}$ in $\langle\cdot, \cdot\rangle_{\mathcal{X}}$ and $\|\cdot\|_{\mathcal{X}}$.

For a linear operator $T$ on a Hilbert space, we denote its domain by $D(T)$. If $T$ is densely defined, the adjoint of $T$ is denoted by $T^{*}$. For linear operators $S$ and $T$ on a Hilbert space, $D(S$ $+T):=D(S) \cap D(T)$ unless otherwise stated.

For a self-adjoint operator $S$ on a Hilbert space, we denote its spectrum and its essential spectrum by $\sigma(S)$ and $\sigma_{\text {ess }}(S)$, respectively. The spectral measure associated with $S$ is denoted by $E_{S}(\cdot)$. If $S$ is bounded below, then we set

$$
E_{0}(S):=\inf \sigma(S)
$$

and call it the ground state energy of $S$. We say that $S$ has a ground state if $E_{0}(S)$ is an eigenvalue of $S$; in this case, each nonzero vector in $\operatorname{ker}\left(S-E_{0}(S)\right)$ is called a ground state of $S$. The number $m(S):=\operatorname{dim} \operatorname{ker}\left(S-E_{0}(S)\right)$ is called the multiplicity of the ground state of $S$.

To describe the Bose field, one uses the boson Fock space over a separable complex Hilbert space $\mathcal{X}$,

$$
\mathcal{F}_{\mathrm{b}}(\mathcal{X}):=\bigoplus_{n=0}^{\infty} \bigotimes_{s}^{n} \mathcal{X}=\left\{\psi \in\left\{\psi^{(n)}\right\}_{n=0}^{\infty} \mid n \geq 0, \psi^{(n)} \in \underset{s}{\otimes} \mathcal{X}, \sum_{n=0}^{\infty}\left\|\psi^{(n)}\right\|^{2}<\infty\right\},
$$

where $\otimes_{s}^{n} \mathcal{X}$ denotes the $n$-fold symmetric tensor product of $\mathcal{X}$ with $\otimes_{s}^{0}:=\mathrm{C}$ (the space of complex numbers). The vector $\Omega:=\{1,0, \ldots\}$ is called the Fock vacuum in $\mathcal{F}_{\mathrm{b}}(\mathcal{X})$.

One of the main objects on $\mathcal{F}_{\mathrm{b}}(\mathcal{X})$ is the annihilation operator $a(f)$ which is a densely defined closed linear operator on $\mathcal{F}_{\mathrm{b}}(\mathcal{X})$, such that for all $\eta=\left\{\eta_{n}\right\}_{n=0}^{\infty} \in D\left(a(f)^{*}\right),\left(a(f)^{*} \eta\right)^{(0)}=0$, and $\left(a(f)^{*} \eta\right)^{(n)}=\sqrt{n} S_{n}\left(f \otimes \eta^{n-1)}\right), n \geq 1$, where $S_{n}$ is the symmetrization operator on $\otimes^{n} \mathcal{X}$. The adjoint $a(f)^{*}$, which is called the creation operator, and the annihilation operator $a(g)$ obey the canonical commutation relations

$$
\left[a(f), a(g)^{*}\right]=\langle f, g\rangle, \quad[a(f), a(g)]=0, \quad\left[a(f)^{*}, a(g)^{*}\right]=0
$$

for all $f, g \in \mathcal{X}$ on the dense subspace,

$$
\mathcal{F}_{0}(\mathcal{X}):=\left\{\eta \in \mathcal{F}_{\mathrm{b}}(\mathcal{X}) \mid \text { there exists a number } n_{0} \text { such that } \eta^{(n)}=0 \text { for all } n \geq n_{0}\right\},
$$

where $[\cdot, \cdot]$ means the commutator.

For every self-adjoint operator $S$ on $\mathcal{X}$, one can define a self-adjoint operator $d \Gamma(S)$, called the second quantization of $S$, by

$$
d \Gamma(S):=\bigoplus_{n=0}^{\infty} S^{(n)},
$$

with $S^{(0)}:=0$ and $S^{(n)}$ is the closure of

$$
\left.(\sum_{j=1}^{n} \underbrace{I \otimes \cdots \otimes \overbrace{S^{j}}^{j} \otimes \cdots}_{n})\right|_{\hat{\otimes}^{n} D(S)}
$$

where $I$ denotes the identity and $\hat{\otimes}^{n} D(S)$ algebraic tensor product of $D(S)$. If $S$ is non-negative, then so is $d \Gamma(S)$. The second quantization $N_{\mathrm{b}}:=d \Gamma(I)$ of the identity is called the number operator.

As the state space of the Dereziński-Gérard model, we take the tensor product Hilbert space, 


$$
\mathcal{F}:=\mathcal{H} \otimes \mathcal{F}_{\mathrm{b}}(\mathcal{K}),
$$

where $\mathcal{H}$ is a separable Hilbert space and $\mathcal{K}=L^{2}\left(\mathbb{R}^{d}\right)$. The Hilbert space $\mathcal{F}$ is identified with the space

$$
\bigoplus_{n=0}^{\infty}\left[\mathcal{H} \otimes \otimes_{s}^{n} \mathcal{K}\right]
$$

and we use this identification freely in what follows.

The subspace $\mathcal{D}_{0}$ of $\mathcal{F}$ is defined as follows:

$$
\mathcal{D}_{0}:=\left\{\psi \in \mathcal{F} \mid \text { there exists an } n_{0} \text { such that, for all } n \geq n_{0}, \psi^{(n)}=0\right\} .
$$

Let $A$ be a self-adjoint operator on $\mathcal{H}$. Let $\omega$ be a non-negative, injective, and self-adjoint operator on $\mathcal{K}$. A typical example of $\omega$ is the multiplication operator by the function $\omega(k)=\sqrt{m^{2}+|k|^{2}}$. Here $m \geq 0$ is the mass of the boson. The function $\omega$ represents a dispersion relation of one free boson associated with the Bose field under consideration.

We define an operator $H_{\mathrm{b}}$ by

$$
H_{\mathrm{b}}:=d \Gamma(\omega)
$$

acting on $\mathcal{F}_{\mathrm{b}}(\mathcal{K})$. The free Hamiltonian of the Dereziński-Gérard model is given by

$$
H_{0}:=A \otimes I+I \otimes H_{\mathrm{b}} .
$$

To define the interaction part of the Dereziński-Gérard model, we introduce an analog $\widetilde{\phi}(v)$ of the usual Segal's field operator for a bounded operator $v$ from $\mathcal{H}$ to $\mathcal{H} \otimes \mathcal{K}$. To do this, we first define the operator $\widetilde{a}^{*}(v)$, which is an analog of the usual creation operator. The domain and the operation of $\widetilde{a}^{*}(v)$ is as follows (Refs. 5 and 10):

$$
\begin{aligned}
& D\left(\widetilde{a}^{*}(v)\right):=\left\{\psi=\left(\psi^{(n)}\right)_{n=0}^{\infty} \in \mathcal{F} \mid \sum_{n=0}^{\infty} n\left\|\left(I_{\mathcal{H}} \otimes S_{n}\right)\left(v \otimes I_{\otimes_{s}^{n-1}} \mathcal{K}\right) \psi^{(n-1)}\right\|^{2}<\infty\right\}, \\
& \left(\widetilde{a}^{*}(v) \psi\right)^{(0)}:=0, \quad\left(\widetilde{a}^{*}(v) \psi\right)^{(n)}:=\sqrt{n}\left(I_{\mathcal{H}} \otimes S_{n}\right)\left(v \otimes I_{\otimes_{s}^{n-1} \mathcal{K}}\right) \psi^{(n-1)}, \quad n \geq 1 .
\end{aligned}
$$

It is easy to see that $D\left(\widetilde{a}^{*}(v)\right) \supset \mathcal{D}_{0}$. Thus the operator $\widetilde{a}^{*}(v)$ is a densely defined. We set

$$
\widetilde{a}(v):=\left(\widetilde{a}^{*}(v)\right)^{*} .
$$

The domain and the operation of $\widetilde{a}(v)$ is as follows:

$$
\begin{gathered}
D(\widetilde{a}(v)):=\left\{\psi=\left(\psi^{(n)}\right)_{n=0}^{\infty} \in \mathcal{F} \mid \sum_{n=0}^{\infty}(n+1)\left\|\left(I_{\mathcal{H}} \otimes S_{n}\right)\left(v^{*} \otimes I_{\otimes_{s}^{n} \mathcal{K}}\right) \psi^{(n+1)}\right\|^{2}<\infty\right\}, \\
(\widetilde{a}(v) \psi)^{(n)}:=\sqrt{n+1}\left(I_{\mathcal{H}} \otimes S_{n}\right)\left(v^{*} \otimes I_{\otimes_{s}^{n} \mathcal{K}}\right) \psi^{(n+1)} .
\end{gathered}
$$

It is easily verified that $\widetilde{a}^{\sharp}(v)\left(\widetilde{a}^{\sharp}=\widetilde{a}\right.$ or $\left.\widetilde{a}^{*}\right)$ is closed and $\mathcal{D}_{0}$ is a core for $\widetilde{a}^{\sharp}(v)$.

We define an analog of Segal's field operator as follows:

$$
\widetilde{\phi}(v):=\frac{1}{\sqrt{2}}\left(\widetilde{a}(v)+\widetilde{a}^{*}(v)\right) .
$$

The total Hamiltonian of the Dereziński-Gérard model is defined as follows: 


$$
H:=H_{0}+\widetilde{\phi}(v) .
$$

Throughout this paper, we assume the following conditions.

(A.1) $A$ is bounded below.

(A.2) (Gap condition) $\Sigma-E_{0}(A)>0$, where $\Sigma:=\inf \sigma_{\text {ess }}(A)$.

For $m>0$, we set

$$
\begin{gathered}
\omega_{m}:=\omega+m, \\
H_{m}:=A \otimes I+I \otimes d \Gamma\left(\omega_{m}\right)+\widetilde{\phi}(v) \\
=H+m I \otimes N_{\mathrm{b}} .
\end{gathered}
$$

(A.3) There exists an $m_{0}>0$, such that if $m \in\left(0, m_{0}\right], H_{m}$ has a ground state.

Remark 1: We make some comments on the assumption (A.3). As stated in Sec I, there are sufficient conditions that the massive Dereziński-Gérard Hamiltonians have a ground state. In spite of these results, the reason why we adopt the assumption (A.3) is to make the present paper self-contained.

To state the following assumption, for $s \in \mathbb{R}$, we introduce a subspace $V_{\omega}^{s}$ of $B(\mathcal{H}, \mathcal{H} \otimes \mathcal{K})$ by

$$
V_{\omega}^{s}:=\left\{v \in B(\mathcal{H}, \mathcal{H} \otimes \mathcal{K}) \mid(I \otimes \omega)^{s} v \in B(\mathcal{H}, \mathcal{H} \otimes \mathcal{K})\right\} .
$$

In what follows, we abbreviate $(I \otimes \omega)^{s} v$ as $\omega^{s} v$.

Remark 2: If $\omega$ is strictly positive, then $V_{\omega}^{s}=B(\mathcal{H}, \mathcal{H} \otimes \mathcal{K})$ for $s \leq 0$.

(A.4) $v \in V_{\omega}^{-1}$.

Remark 3: One can easily see that $v \in V_{\omega}^{-1}$ implies $v \in V_{\omega}^{-1 / 2}$. The referee remarked this fact to the author. The author is thankful to the referee for it.

The following theorem is the main theorem of the present paper.

Theorem 2.1: (Existence of a ground state for the massless case) Assume (A.1)-(A.4). Suppose that

$$
\frac{1}{4\left(\Sigma-E_{0}(H)\right)^{2}}\|v\|^{2}\left\|\omega^{-1} v\right\|^{2}+\frac{\left\|\omega^{-1} v\right\|^{2}}{2}<1 .
$$

Then the massless Dereziński-Gérard model has a ground state.

\section{ELEMENTARY RESULTS FOR THE DEREZIŃSKI-GÉRARD MODEL}

In this section, we establish some elementary results for the Dereziński-Gérard model. These results hold independent of whether $H$ is massive or massless. We use these results freely throughout this paper.

Proposition 3.1: (Energy bound for the Dereziński-Gérard Hamiltonian)

$$
E_{0}(H) \leq E_{0}(A) \text {. }
$$

Proof: Let $u \in D(A)$ with $\|u\|=1$. Then, by the variational principle,

$$
E_{0}(H) \leq\langle u \otimes \Omega, H u \otimes \Omega\rangle=\langle u, A u\rangle .
$$

Using the variational principle again, we obtain the desired result.

Proposition 3.1 means that the interaction does not raise the ground state energy in the Dereziński-Gérard model.

Let $v \in B(\mathcal{H}, \mathcal{H} \otimes \mathcal{K})$. The operator $v$ corresponds to the $B(\mathcal{H})$-valued function $v(\cdot)$ on $\mathbb{R}^{d}$ defined by 


$$
v(k) \xi:=(v \xi)(k), \quad \xi \in \mathcal{H}, \quad k \in \mathbb{R}^{d} .
$$

As commutation relations for $\widetilde{a}^{\sharp}(v)$, the following commutation relations hold.

Theorem 3.2: (Reference 1) Let $v_{1}, v_{2} \in B(\mathcal{H}, \mathcal{H} \otimes \mathcal{K})$. If $\left[v_{1}(k)^{*}, v_{2}\left(k^{\prime}\right)\right]=0$ for a.e. $k, k^{\prime}$ $\in \mathbb{R}^{d}$, then the following commutation relations hold on $\mathcal{D}_{0}$ :

$$
\left[\widetilde{a}\left(v_{1}\right), \widetilde{a}^{*}\left(v_{2}\right)\right]=v_{1}^{*} v_{2} \otimes I, \quad\left[\widetilde{a}\left(v_{1}\right), \widetilde{a}\left(v_{2}\right)\right]=0, \quad\left[\widetilde{a}^{*}\left(v_{1}\right), \widetilde{a}^{*}\left(v_{2}\right)\right]=0 .
$$

The following relative boundedness of $\widetilde{a}(v)$ is fundamental.

Theorem 3.3: (Reference 6) For $v \in V_{\omega}^{-1 / 2}, D(\widetilde{a}(v)) \supset D\left(I \otimes H_{\mathrm{b}}^{1 / 2}\right)$, and, for $\psi \in D\left(I \otimes H_{\mathrm{b}}^{1 / 2}\right)$,

$$
\|\widetilde{a}(v) \psi\| \leq\left\|\omega^{-1 / 2} v\right\|\left\|I \otimes H_{\mathrm{b}}^{1 / 2} \psi\right\| .
$$

Substituting $I$ into $\omega$ in the above theorem, we obtain the following relative boundedness of $\widetilde{a}(v)$ with respect to $I \otimes N_{\mathrm{b}}^{1 / 2}$.

Corollary 3.4: For $v \in B(\mathcal{H}, \mathcal{H} \otimes \mathcal{K}), D(\widetilde{a}(v)) \supset D\left(I \otimes N_{\mathrm{b}}^{1 / 2}\right)$, and, for $\psi \in D\left(I \otimes N_{\mathrm{b}}^{1 / 2}\right)$,

$$
\|\widetilde{a}(v) \psi\| \leq\|v\|\left\|I \otimes N_{\mathrm{b}}^{1 / 2} \psi\right\| .
$$

Theorem 3.3 and the Kato-Rellich theorem imply the self-adjointness of the total Hamiltonian.

Theorem 3.5: (Self-adjointness of the total Hamiltonian) Let $v \in V_{\omega}^{-1 / 2}$. Then the total Hamiltonian $H$ is self-adjoint and bounded below. Moreover, any core of $H_{0}$ is also a core of $H$.

\section{SOME LEMMAS FOR THE MASSIVE CASE}

We devote this section to the preparation of some lemmas needed in the proof of Theorem 2.1. In this section, we assume that the operator $\omega$ is strictly positive.

Combining Lemma 4.2 in Ref. 2 with Theorem VIII.4 in Ref. 11.

Lemma 4.1: Let $\left\{f_{l}\right\} \subset D\left(\omega^{1 / 2}\right)$ be a complete orthonormal system of $\mathcal{K}$. Then for all $\psi$ $\in D\left(I \otimes H_{\mathrm{b}}\right)$,

$$
\sum_{l=1}^{\infty}\left\langle I \otimes a\left(\omega^{-1 / 2} f_{l}\right) \psi, I \otimes a\left(\omega^{1 / 2} f_{l}\right) \psi\right\rangle=\left\langle\psi, I \otimes N_{\mathrm{b}} \psi\right\rangle
$$

We introduce a subspace $\mathcal{F}_{\text {fin }}(\omega)$ of $\mathcal{F}_{\mathrm{b}}(\mathcal{K})$ and a subspace $\mathcal{D}_{\omega}$ of $\mathcal{F}$ as follows:

$$
\begin{gathered}
\mathcal{F}_{\text {fin }}(\omega):=\mathcal{L}\left\{\Omega, a\left(g_{1}\right)^{*} \cdots a\left(g_{n}\right)^{*} \Omega \mid n \geq 1, g_{1} \cdots g_{n} \in D(\omega)\right\}, \\
\mathcal{D}_{\omega}:=D(A) \hat{\otimes} \mathcal{F}_{\text {fin }}(\omega) .
\end{gathered}
$$

For $f \in \mathcal{K}$, we define a bounded operator $v_{f}$ from $\mathcal{H}$ into $\mathcal{H} \otimes \mathcal{K}$ by

$$
v_{f} \xi:=\xi \otimes f, \quad \xi \in \mathcal{H}
$$

Note that

$$
\widetilde{a}\left(v_{f}\right)=I \otimes a(f) .
$$

Lemma 4.2: For $f \in D(\omega)$, we define

$$
T(f):=I \otimes a(\omega f)+\frac{1}{\sqrt{2}} v_{f}^{*} v \otimes I .
$$

Let $\psi_{E}$ be an eigenvector of $H$ with eigenvalue E. Then, $\psi_{E} \in D(T(f)) \cap D(I \otimes a(f)), I \otimes a(f) \psi_{E}$ $\in D(H)$, and $(H-E) I \otimes a(f) \psi_{E}=-T(f) \psi_{E}$.

Proof: For $\psi \in \mathcal{D}_{\omega}$, 


$$
\begin{aligned}
I \otimes a(f)^{*} H \psi & =\left[I \otimes a(f)^{*}, H\right] \psi+H I \otimes a(f)^{*} \psi \\
& =\left\{\left[I \otimes a(f)^{*}, I \otimes H_{\mathrm{b}}\right]+\left[I \otimes a(f)^{*}, \widetilde{\phi}(v)\right]\right\} \psi+H I \otimes a(f)^{*} \psi \\
& =-I \otimes a(\omega f)^{*} \psi-\frac{1}{\sqrt{2}} v^{*} v_{f} \otimes I \psi+H I \otimes a(f)^{*} \psi \\
& =-T(f)^{*} \psi+H I \otimes a(f)^{*} \psi .
\end{aligned}
$$

Therefore, we have

$$
\begin{aligned}
\left\langle I \otimes a(f) \psi_{E}, H \psi\right\rangle & =\left\langle\psi_{E}, I \otimes a(f)^{*} H \psi\right\rangle \\
& =\left\langle\psi_{E},-T(f)^{*} \psi\right\rangle+\left\langle\psi_{E}, H I \otimes a(f)^{*} \psi\right\rangle \\
& =-\left\langle T(f) \psi_{E}, \psi\right\rangle+\left\langle E \psi_{E}, I \otimes a(f)^{*} \psi\right\rangle \\
& =-\left\langle T(f) \psi_{E}, \psi\right\rangle+E\left\langle I \otimes a(f) \psi_{E}, \psi\right\rangle .
\end{aligned}
$$

Since $\mathcal{D}_{\omega}$ is a core of $H$, the above equality holds for $\psi \in D(H)$. Hence, we obtain

$$
I \otimes a(f) \psi_{E} \in D(H)
$$

and

$$
(H-E) I \otimes a(f) \psi_{E}=-T(f) \psi_{E}
$$

The next two lemmas are key lemmas to prove the main theorem.

Lemma 4.3: Let $\widetilde{\psi}_{0}$ be a normalized ground state of $H$. Then

$$
\left\langle\tilde{\psi}_{0}, I \otimes N_{\mathrm{b}} \tilde{\psi}_{0}\right\rangle \leq \frac{\left\|\omega^{-1} v\right\|^{2}}{2} .
$$

Proof: For $f \in D(\omega)$, by Lemma 4.2, we have

$$
\begin{aligned}
0 \leq & \left\langle I \otimes a(f) \tilde{\psi}_{0},\left(H-E_{0}(H)\right) I \otimes a(f) \tilde{\psi}_{0}\right\rangle \\
= & -\left\langle I \otimes a(f) \tilde{\psi}_{0}, T(f) \tilde{\psi}_{0}\right\rangle \\
= & -\left\langle I \otimes a(f) \tilde{\psi}_{0}, I \otimes a(\omega f) \tilde{\psi}_{0}\right\rangle \\
& -\frac{1}{\sqrt{2}}\left\langle I \otimes a(f) \tilde{\psi}_{0},\left(v_{f}^{*} v \otimes I\right) \tilde{\psi}_{0}\right\rangle .
\end{aligned}
$$

Substituting $\omega^{-1 / 2} f_{l}$ into $f$, we have

$$
\left\langle I \otimes a\left(\omega^{-1 / 2} f_{l}\right) \tilde{\psi}_{0}, I \otimes a\left(\omega^{1 / 2} f_{l}\right) \tilde{\psi}_{0}\right\rangle \leq-\frac{1}{\sqrt{2}}\left\langle I \otimes a\left(\omega^{-1 / 2} f_{l}\right) \tilde{\psi}_{0},\left(v_{\omega^{-1 / 2} f_{l}}^{*} v \otimes I\right) \tilde{\psi}_{0}\right\rangle .
$$

Summing up with respect to $l$, we have

$$
\sum_{l=1}^{\infty}\left\langle I \otimes a\left(\omega^{-1 / 2} f_{l}\right) \tilde{\psi}_{0}, I \otimes a\left(\omega^{1 / 2} f_{l}\right) \tilde{\psi}_{0}\right\rangle=\left\langle\tilde{\psi}_{0}, I \otimes N_{\mathrm{b}} \tilde{\psi}_{0}\right\rangle
$$

by Lemma 4.1.

On the other hand, 


$$
\begin{aligned}
\sum_{l=1}^{\infty}\left\langle I \otimes a\left(\omega^{-1 / 2} f_{l}\right) \tilde{\psi}_{0},\left(v_{\omega^{-1 / 2} f_{l}}^{*} v \otimes I\right) \tilde{\psi}_{0}\right\rangle & =\sum_{l=1}^{\infty}\left\langle\widetilde{a}\left(v_{\omega^{-1 / 2} f_{l}}\right) \tilde{\psi}_{0},\left(v_{\omega^{-1 / 2} f_{l}}^{*} v \otimes I\right) \tilde{\psi}_{0}\right\rangle \\
& =\sum_{l=1}^{\infty}\left\langle\left(v^{*} v_{\omega^{-1 / 2} f_{l}} \otimes I\right) \widetilde{a}\left(v_{\omega^{-1 / 2} f_{l}}\right) \tilde{\psi}_{0}, \widetilde{\psi}_{0}\right\rangle \\
& =\sum_{l=1}^{\infty}\left\langle\widetilde{a}\left(v_{\omega^{-1 / 2} f_{l}} v_{\omega^{-1 / 2} f_{l}}^{*} v\right) \tilde{\psi}_{0}, \tilde{\psi}_{0}\right\rangle=\left\langle\widetilde{a}\left(\omega^{-1} v\right) \tilde{\psi}_{0}, \tilde{\psi}_{0}\right\rangle .
\end{aligned}
$$

In the last equality, we used the fact that

$$
\sum_{l=1}^{\infty} v_{\omega^{-1 / 2} f_{l}} v_{\omega^{-1 / 2} f_{l}}^{*}=(I \otimes \omega)^{-1} .
$$

By Corollary 3.4, we have

$$
\left|\left\langle\widetilde{a}\left(\omega^{-1} v\right) \tilde{\psi}_{0}, \tilde{\psi}_{0}\right)\right\rangle \mid \leq\left\|\omega^{-1} v\right\|\left\|I \otimes N_{\mathrm{b}}^{1 / 2} \widetilde{\psi}_{0}\right\|=\left\|\omega^{-1} v\right\|\left\langle\tilde{\psi}_{0}, I \otimes N_{\mathrm{b}} \tilde{\psi}_{0}\right\rangle^{1 / 2} .
$$

Thus, (4.1)-(4.4) imply that

$$
\left\langle\tilde{\psi}_{0}, I \otimes N_{\mathrm{b}} \tilde{\psi}_{0}\right\rangle \leq \frac{\left\|\omega^{-1} v\right\|^{2}}{2} .
$$

This proves the lemma.

Let $E_{A}$ be the spectral measure associated with $A$. We set $\left\{\mu_{r}\right\}_{r=1}^{q}:=\sigma(A) \cap\left[E_{0}(A), \Sigma\right)(q \leq \infty)$. We define an operator $Q_{r}$ on $\mathcal{H}$ by $Q_{r}:=E_{A}\left(\left[E_{0}(A), \mu_{r}\right)\right)$ and set $Q_{r}^{\perp}:=I-Q_{r}$. We denote the orthogonal projection onto the subspace spanned by the Fock vacuum $\Omega$ by $P_{\Omega}$.

Lemma 4.4: Let $\widetilde{\psi}_{0}$ be as above. Then

$$
\left\langle\tilde{\psi}_{0}, Q_{r}^{\perp} \otimes P_{\Omega} \tilde{\psi}_{0}\right\rangle \leq \frac{1}{4\left(\mu_{r+1}-E_{0}(H)\right)^{2}}\|v\|^{2}\left\|\omega^{-1} v\right\|^{2} .
$$

Proof: Simple calculations show that

$$
Q_{r}^{\perp} \otimes P_{\Omega} H=Q_{r}^{\perp} A \otimes P_{\Omega}+\frac{1}{\sqrt{2}} Q_{r}^{\perp} \otimes P_{\Omega} \widetilde{a}(v)
$$

and

$$
Q_{r}^{\perp} \otimes P_{\Omega}\left(H-E_{0}(H)\right) \tilde{\psi}_{0}=0 .
$$

Thus, we have

$$
\left\langle\tilde{\psi}_{0}, Q_{r}^{\perp}\left(A-E_{0}(H)\right) \otimes P_{\Omega} \tilde{\psi}_{0}\right\rangle=-\frac{1}{\sqrt{2}}\left\langle Q_{r}^{\perp} \otimes P_{\Omega} \tilde{\psi}_{0}, \widetilde{a}(v) \tilde{\psi}_{0}\right\rangle .
$$

By using the spectral theorem, we have

$$
\left\langle\tilde{\psi}_{0}, Q_{r}^{\perp}\left(A-E_{0}(H)\right) \otimes P_{\Omega} \tilde{\psi}_{0}\right\rangle \geq\left(\mu_{r+1}-E_{0}(H)\right)\left\langle\widetilde{\psi}_{0}, Q_{r}^{\perp} \otimes P_{\Omega} \tilde{\psi}_{0}\right\rangle .
$$

Hence, we have 


$$
\left\langle\tilde{\psi}_{0}, Q_{r}^{\perp} \otimes P_{\Omega} \tilde{\psi}_{0}\right\rangle \leq \frac{1}{\sqrt{2}\left(\mu_{r+1}-E_{0}(H)\right)}\left\|Q_{r}^{\perp} \otimes P_{\Omega} \tilde{\psi}_{0}\right\|\left\|\tilde{a}(v) \tilde{\psi}_{0}\right\| .
$$

Therefore, we obtain

$$
\left\langle\widetilde{\psi}, Q_{r}^{\perp} \otimes P_{\Omega} \widetilde{\psi}_{0}\right\rangle \leq \frac{1}{2\left(\mu_{r+1}-E_{0}(H)\right)^{2}}\left\|\widetilde{a}(v) \tilde{\psi}_{0}\right\|^{2} .
$$

By applying Lemma 4.3 and the inequality

$$
\left\|\widetilde{a}(v) \widetilde{\psi}_{0}\right\|^{2} \leq\|v\|^{2}\left\|I \otimes N_{\mathrm{b}}^{1 / 2} \widetilde{\psi}_{0}\right\|^{2},
$$

we obtain

$$
\left\langle\tilde{\psi}_{0}, Q_{r}^{\perp} \otimes P_{\Omega} \tilde{\psi}_{0}\right\rangle \leq \frac{1}{4\left(\mu_{r+1}-E_{0}(H)\right)^{2}}\|v\|^{2}\left\|\omega^{-1} v\right\|^{2} .
$$

\section{EXISTENCE OF A GROUND STATE OF THE MASSLESS DEREZIŃSKI-GÉRARD MODEL}

This section is the main part of the present paper. We shall prove the existence of a ground state of the massless Dereziński-Gérard model by applying Lemma 5.1 below. Lemma 5.1 was established by Arai and Hirokawa in Ref. 2. Since then, this lemma has been widely used to prove the existence of a ground state of massless models by many researchers. ${ }^{2,3,8,9}$

Lemma 5.1: (Reference 2) Let $A_{n}$ and $A$ be self-adjoint operators on a Hilbert space $\mathcal{H}$ which are bounded below. Suppose that $A_{n}$ has a ground state $\psi_{n}, E_{0}\left(A_{n}\right)$ converges to $E_{0}(A)$, and $A_{n}$ and $A$ have a common core $\mathcal{D}$, such that $A_{n} \psi$ converges to $A \psi$ for all $\psi \in \mathcal{D}$. If there exists a nonzero vector $\psi_{0} \in \mathcal{H}$ such that $\psi_{n}$ converges to $\psi_{0}$ weakly, then $\psi_{0}$ is a ground state of $A$.

Let $H$ be the Dereziński-Gerrard Hamiltonian and $H_{m}$ be the operator defined by (2.1). By the assumption (A.3), there exists a normalized ground state $\psi(m)$ of $H_{m}$. By the weak compactness of the closed unit ball of the Hilbert space $\mathcal{F}$, there exists $\psi_{0} \in \mathcal{F}$ and a subsequence $\left\{\psi\left(m_{j}\right)\right\}$ which converges to $\psi_{0}$ weakly with $m_{j} \backslash 0$. To apply Lemma 5.1 above, we must check that (1) $\psi_{0}$ $\neq 0$, (2) $E_{0}\left(H_{m}\right)$ converges to $E_{0}(H)$ as $m \rightarrow 0$, and (3) the existence of a common core of $H$ and $H_{m}$ on which $H_{m}$ converges to $H$ strongly.

Recall the subspace $\mathcal{D}_{\omega}$ introduced in Sec. IV. By the general theory of tensor product of operators, $\mathcal{D}_{\omega}$ is a core of $H_{0}$. This fact, Theorem 3.5 and Eq. (2.1) imply the following fact.

Lemma 5.2: $\mathcal{D}_{\omega}$ is a common core of $H$ and $H_{m}$ and $H_{m} \psi \rightarrow H \psi$ for all $\psi \in \mathcal{D}_{\omega}$.

Lemma 5.3:

$$
E_{0}\left(H_{m}\right) \rightarrow E_{0}(H) .
$$

Proof: By Lemma 5.2 above, $H_{m}$ converges to $H$ in the strong resolvent sense. Hence

$$
\limsup _{m \rightarrow 0} E_{0}\left(H_{m}\right) \leq E_{0}(H) .
$$

On the other hand,

$$
H_{m} \geq H \text { for all } m>0 \text {. }
$$

Therefore,

$$
E_{0}\left(H_{m}\right) \geq E_{0}(H) \text { for all } m>0 \text {. }
$$

So we obtain 


$$
\liminf _{m \rightarrow 0} E_{0}\left(H_{m}\right) \geq E_{0}(H) .
$$

Thus, we have the desired result.

In the proof of Theorem 2.1, we use the following operator inequality.

Lemma 5.4: (Reference 2)

$$
Q_{r} \otimes P_{\Omega} \geq I-I \otimes N_{\mathrm{b}}-Q_{r}^{\perp} \otimes P_{\Omega} .
$$

We are now ready to prove Theorem 2.1 .

Proof of Theorem 2.1: The conditions (2) and (3) above correspond to Lemma 5.3 and Lemma 5.2 , respectively. So, we have only to check the condition (1).

By Lemmas 4.3, 4.4, and 5.4, we have

$$
\begin{aligned}
\left\langle\psi\left(m_{j}\right), Q_{r} \otimes P_{\Omega} \psi\left(m_{j}\right)\right\rangle & \geq 1-\left\langle\psi\left(m_{j}\right), I \otimes N_{\mathrm{b}} \psi\left(m_{j}\right)\right\rangle-\left\langle\psi\left(m_{j}\right), Q_{r}^{\perp} \otimes P_{\Omega} \psi\left(m_{j}\right)\right\rangle \\
& \geq 1-\frac{\left\|\omega_{m_{j}}^{-1} v\right\|^{2}}{2}-\frac{1}{4\left(\mu_{r+1}-E_{0}(H)\right)^{2}}\|v\|^{2}\left\|\omega_{m_{j}}^{-1} v\right\|^{2} \\
& \geq 1-\frac{\left\|\omega^{-1} v\right\|^{2}}{2}-\frac{1}{4\left(\mu_{r+1}-E_{0}(H)\right)^{2}}\|v\|^{2}\left\|\omega^{-1} v\right\|^{2} .
\end{aligned}
$$

Tending $m_{j} \rightarrow \infty$, we have

$$
\left\langle\psi_{0}, Q_{r} \otimes P_{\Omega} \psi_{0}\right\rangle \geq 1-\frac{\left\|\omega^{-1} v\right\|^{2}}{2}-\frac{1}{4\left(\mu_{r+1}-E_{0}(H)\right)^{2}}\|v\|^{2}\left\|\omega^{-1} v\right\|^{2} .
$$

The right hand side of the above inequality converges to

$$
1-\frac{\left\|\omega^{-1} v\right\|^{2}}{2}-\frac{1}{4\left(\Sigma-E_{0}(H)\right)^{2}}\|v\|^{2}\left\|\omega^{-1} v\right\|^{2}
$$

as $r \rightarrow \infty$. Therefore, for sufficiently large $r$,

$$
\left\langle\psi_{0}, Q_{r} \otimes P_{\Omega} \psi_{0}\right\rangle>0
$$

Hence $\psi_{0} \neq 0$. By applying Lemma 5.1, we have the desired result.

\section{ACKNOWLEDGMENTS}

The author would like to express his deep gratitude to Professor Arai of Hokkaido University. He carefully read the whole manuscript of the present paper and gave him many valuable suggestions throughout the paper. The author could not complete the paper without his support. He is also grateful to Professor Iso of Kyoto University for encouragement. He also thanks lecturer Kubo of Kyoto University and Mr. Kakuma for fruitful discussions. He also appreciates referee's commment very much, for they help us to improve the quality of our paper considerably.

\footnotetext{
${ }^{1}$ Ammari, Z., "Asymptotic completeness for a renormalized nonrelativistic Hamiltonian in quantum field theory: the Nelson model," Math. Phys., Anal. Geom. 3, 217 (2000).

2 Arai, A. and Hirokawa, M., "On the existence and uniqueness of ground states of a generalized spin-boson model," J. Funct. Anal. 151, 455 (1997)

${ }^{3}$ Arai, A. and Hirokawa, M., "Ground states of a general class of quantum field Hamiltonians," Rev. Math. Phys. 12, 1085 (2000).

${ }^{4}$ Bruneau, L. and Dereziński, J., "Pauli-Fierz Hamiltonians defined as quadratic forms," Rep. Math. Phys. 54, 169 (2004).

${ }^{5}$ Dereziński, J. and Gérard, C., "Asymptotic completeness in quantum field theory. Massive Pauli-Fierz Hamiltonians," Rev. Math. Phys. 11, 383 (1999).

${ }^{6}$ Dereziński, J. and Jakšić, V., "Spectral theory of Pauli-Fierz operators," J. Funct. Anal. 180, 243 (2001).

${ }^{7}$ Georgescu, V., Gérard, C., and Møller, J. S., "Spectral theory of massless Pauli-Fierz models," Commun. Math. Phys. 249, 29 (2004)
} 
${ }^{8}$ Gérard, C., "On the existence of ground states for massless Pauli-Fierz Hamiltonians," Ann. Henri Poincare 1, 443 (2000).

${ }^{9}$ Hiroshima, F., "Ground states and spectrum of quantum electrodynamics of nonrelativistic particles," Trans. Am. Math. Soc. 353, 4497 (2001).

${ }^{10}$ Miyao, T. and Sasaki, I., "Stability of discrete ground state," Hokkaido Math. J. 34, 689 (2005).

${ }^{11}$ Reed, M. and Simon, B., Methods of Modern Mathematical Physics. I. Functional Analysis (Academic, New York, 1972). 